\title{
Integration of Fuzzy Reasoning approach (FRA) and Fuzzy Analytic Hierarchy Process (FAHP) for Risk Assessment in Mining Industry
}

\author{
Shikha Verma, Sharad Chaudhri \\ Nagpur University (India) \\ shikhaverma2108@gmail.com,sschaudharipati@@rediffmail.com
}

Received: September 2013

Accepted: July 2014

\section{Abstract:}

Purpose: Mining industry has always been known for its unsafe working environment. This industry is one of the most hazard prone industries. To maintain safety in workplace timely assessment of risk associated with different operations performed to extract ore from the ore body has become necessity. To serve the said purpose, present work demonstrates a robust hybrid risk assessment approach for mining industry.

Design/Methodology: Accident data from 1995 to 2012 is reviewed to identify hazards contributed to negative outcomes. The FRA approach is implemented to evaluate the risk levels associated with identified hazard factors. Thereafter AHP pairwise comparison matrix is developed to obtain priority weights for the hazard factors. Final priority of hazards based on severity of level of risk associated with them is obtained considering the outcome of FRA approach in terms of risk score for the hazards, combined with the priority weights obtained from AHP technique.

Findings: Defuzzified FAHP weight of hazard factors, this weight gives priority sequence of hazards to be considered for development of plan of mitigation.

Originality/Value: Risk assessment is a requirement of the Occupational Health and Safety Act 2000 (Section $7 \&$ 8). The data required to assess the risk is uncertain, and in such case fuzzy approach is well suited to process the data and get the crisp output. The output of fuzzy 
approach is made robust with its integration to AHP. In this way FAHP can be used as robust technique for risk assessment in this industry and this technique develops an efficient safety management system for the achievement of goal to develop the workplace with zero accident, which many other countries have already achieved.

Keywords: risk assessment, hazard, fuzzy, AHP, mining, FAHP, FRA

\section{Introduction}

Workers in any industry get adversely affected with hazardous working environment. As a result of this, injuries, fatalities, loss of man hours, loss of work days come into visibility. Hazard is a source with potential to cause harm. Risk is chance that how likely hazard will give rise to unacceptable consequences. Risk assessment is a technique which helps mine operators to identify low, medium, high levels of risk associated with hazards. This will help them to prioritize the hazards based upon the levels of risk associated with them, so that hazard with highest potential to cause harm can get mitigate and safe work environment can be developed. Risk assessment is a requirement of the Occupational Health and Safety Act 2000 (section 7 \& 8). According to DGMS (Directorate General of Mining Safety) annual report (2012), from 2002 to 2011 in India 620 people were killed and 1070 people got seriously injured in metalliferrous mines. These figures reveal the fact that efforts can be taken in the area of safety management for mining industries. Some of the major causes of injuries identified in the mining sector are fall of roof, fall of sides, rope haulages, winding in shaft, explosives, electricity, dust/gas etc (DGMS 2010, 2012). The causes mentioned above are the hazards with potential to cause harm to the human life. Assessment of risk associated with these hazards will help managers and safety officers to take decision regarding timely mitigation of the adverse impacts and create a safe work environment which will ultimately help the organization in the enhancement of productivity. In this paper a integrated approach is proposed which can be used for the assessment of risk in the mining sector. This approach comes under the category of Multi criteria decision making (MCDM) method, it refers to making decision in the presence of multiple and conflicting criteria (Tang, Tzeng \& Wang, 2000; Huang, An \& Baker, 2005; Saaty, 1980; Mikhaiov, 2004; An, Huang \& Baker, 2007; Venkatrao, 2013; Coyle G., 2004; Vahdani, Mousavi \& Tavakkoli-Moghaddam, 2011; Ross, 2010; MATLAB help). MCDM is used by many researchers in different areas like, supply chain management, Energy management, chemical engineering, aviation industry, nuclear industry, health and safety management, drought disasters, fabrication industry (MATLAB help; Ross, 2010; Tang et al., 2000; Huang et al., 2005; Mikhailov, 2004; An et al., 2007). The approach proposed in this work is fuzzy Analytic Hierarchy Process (AHP). 


\section{Criteria for Risk Evaluation in Mining Industry}

The criteria selection for the evaluation of risk levels in mining industry involves detailed study of accident data for the span of time under consideration. The data for accident gives information regarding the hazards involved in developing unacceptable consequences. The evaluation of levels of risk associated with these hazard gives idea about how, to what extent and with what priority the mitigation plan for the hazards to be prepared and implement. This gives chance of timely treatment of most risky hazard, which ultimately will prevent any mishap to happen. From the accident data reviewed for the present work from 1995 to 2011, hazard factors/sub criteria identified are- Ground movement, winding in shaft, transportation by machinery, machinery other than transportation, explosives, electricity, dust/gas, falls other than ground (DGMS 2010, 2012). The above mentioned hazards are evaluated on the basis of three criteria, consequence of severity, level of exposure, probability of occurrence (Pathak \& Sen, 2001; Paliwal \& Jain, 2001; Tripathy \& Patra, 1998; Lakshminarayan \& Singh, 2000).

Steps involved for the identification of the criteria is shown in Figure 1 and Figure 2 shows the application of FAHP (proposed approach).

\section{FAHP (Proposed Approach)}

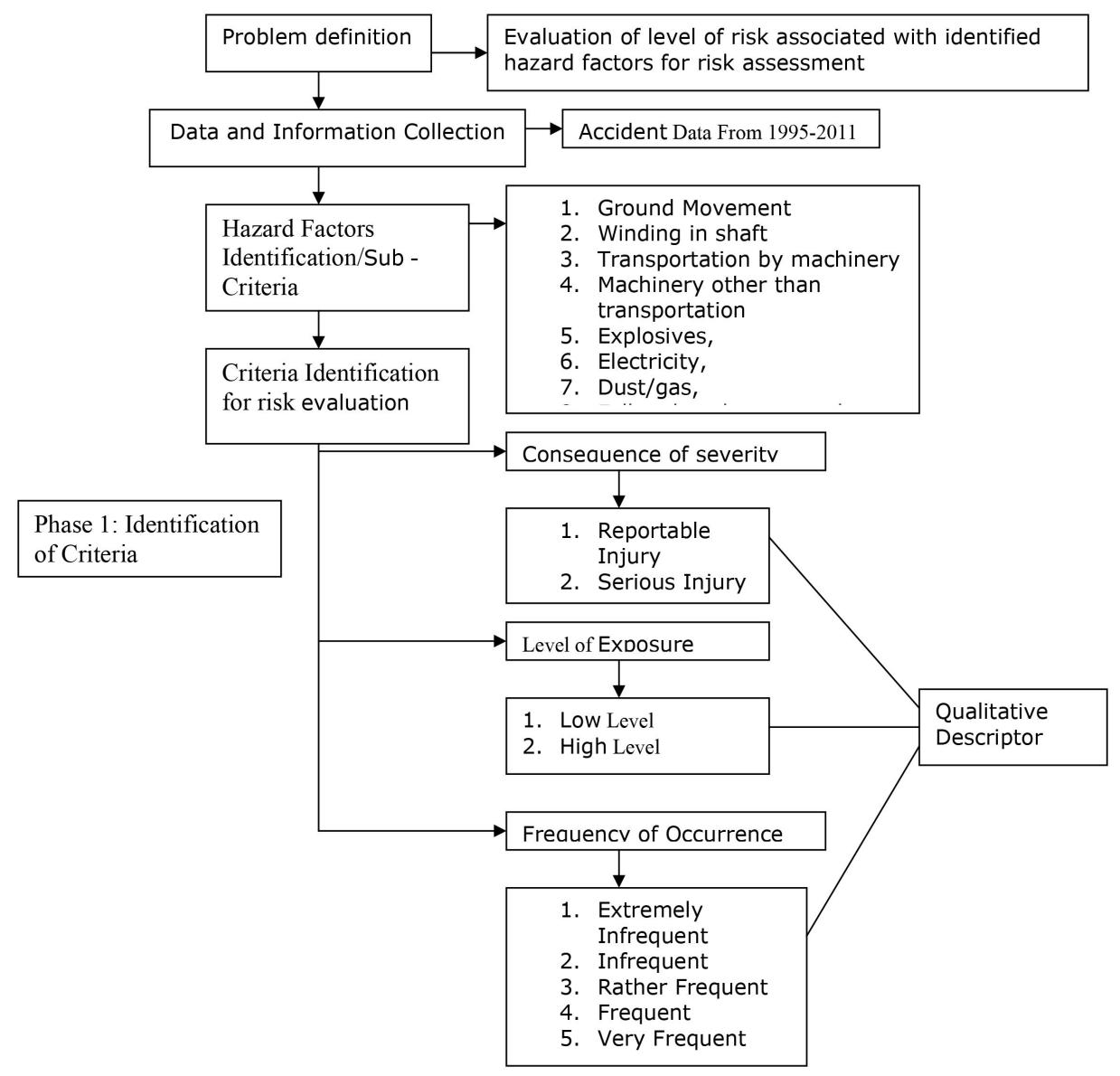

Figure 1. Criteria identification 


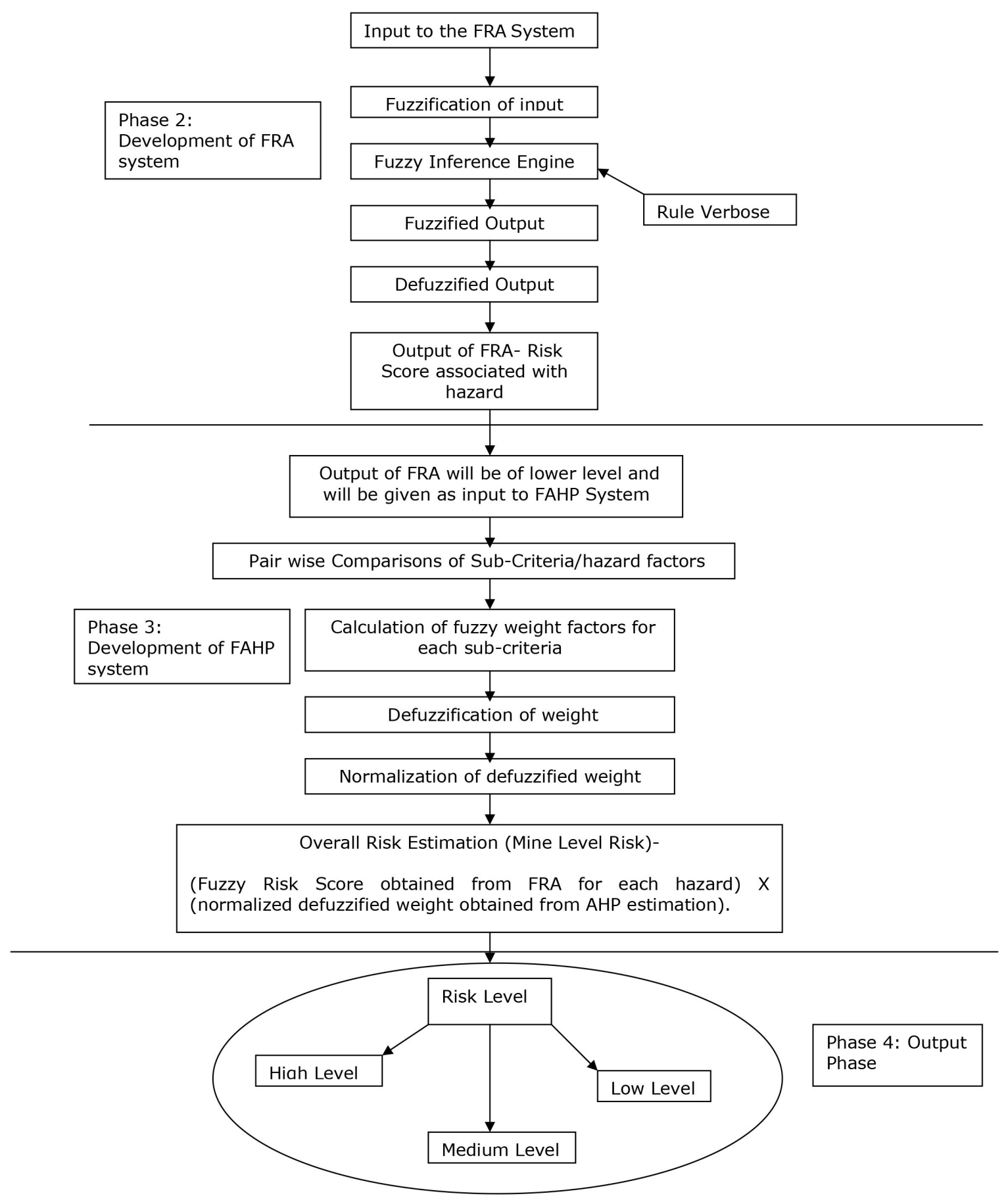

Figure 2. FAHP System

\subsection{Fuzzy Reasoning Approach (FRA)}

FRA stands for fuzzy reasoning approach. This approach is best suited for those cases where availability of data is highly uncertain (MATLAB help; Ross, 2010; Tang et al., 2000; Huang et al., 2005; Mikhailov, 2004; An et al., 2007; An, Chen, \& Baker, 2011; Mohammadi \& Meech, 2013; Vahdani et al., 2011; Venkatrao, 2013, Buyukozkan \& Cifci, 2012a, 2012b; Chen \& Yang, 2011, Khalil, Abdou, Mansour, Farag \& Ossman, 2012; Rouhani, Ghazanfari \& Jaari, 2012; Wang, Fan \& Wang, 2010; Wang \& Lee, 2007). Either data is over available or scanty. 
FRA compensates the part of any analysis which needs human perception and expertise to derive any conclusion based upon the data available. This is done with the help of rule base which is developed based upon the identified criteria. All the necessary steps required for evaluation of risk levels associated with identified sub criteria/hazard factors, using fuzzy rule base reasoning approach consists of following four major components.

\subsubsection{Identify Causes/Factors}

In this component all the potential causes which can cause a mishap are identified. This can be done with the help of accident data maintained by the organization at mine level as well as central level for all the mines owned by the organization. Annual report generated by Directorate General of Mining Safety (DGMS) also provides year wise, places wise, cause wise, accident data for both metalliferrous and non metalliferrous mines.

\subsubsection{Identification of fuzzy input and fuzzy output variables}

In this component inputs to the FRA system and the expected output from FRA system is defined. For the present work of assessment of risk levels associated with the identified hazards, each potential hazard is evaluated on the basis of three criteria namely frequency of occurrence (FO), consequence of severity (CS), exposure level (E). For the evaluation of risk level for any hazard, accident data will be input to the FRA system, the system will analyze the input based on above mentioned criteria. Thereafter the output in term of risk score is obtained from FRA system. This risk score is then fit into the scale of risk level (RL) to know the level of risk associated with the hazard.

In FRA the yardsticks for each criterion are developed. These yardsticks are qualitative in nature. To develop the yardstick it is necessary to assign membership function for each of the qualitative descriptor of the criteria to understand the degree of membership of the input given to the system with the space. Similarly yardstick for output expected from the system is developed. This yardstick will also have detailed description of qualitative descriptor with assigned membership function (MATLAB help; Ross, 2010; Tang et al., 2000; An et al., 2007; An et al., 2011).

\subsubsection{Construction of fuzzy rule base}

The number of rules in the fuzzy rule base depends upon the count of qualitative descriptor considered for each of the identified criteria (Huang et al., 2005; An et al., 2007; An et al., 
2011). The if-then rule statements are used to formulate the conditional statement that comprises fuzzy logic. Any fuzzy rule appears in the following form:

if $x$ is $A$ then $y$ is $B$

If $X$ and $Y$ is the universe of discourse and its elements are denoted by $x$ and $y$ respectively. $A$ and $B$ are linguistic variables defined by fuzzy sets on the ranges $X$ and $Y$, respectively. The if part of the rule " $x$ is $A$ " is known as 'antecedent' and the later part of the rule " $y$ is $B$ " is called the 'consequent'. For the present work an example of a rule is

- If Exposure is Low, Frequency of Occurrence is Extremely Infrequent and Consequence of Severity is Reportable then Risk Level is Low.

The input to an if-then rule for the above example is current value for the input variableExposure, Frequency of Occurrence, Consequence of Severity, these are decided from the accident database. The output is entire fuzzy set, for the above rule it is Low. This set will be later defuzzified and crisp output in terms of risk score is obtained (MATLAB help; Ross, 2010; Mohammadi \& Meech, 2013; Chen, Lien, Liu, Liou, Tzeng \& Yang, 2011; Dağdeviren, Yavuz \& Kılınç, 2009; Razani, Yazdani-Chamzini \& Yakhchali, 2013). If the antecedent is in more than one part as shown above in present case then fuzzy operators are used. There are two built in fuzzy operators OR and AND that can be used in such cases. The consequent specifies a fuzzy set to be assigned to the output. Then the implication of consequent is done. The implication function modifies that fuzzy set to the degree specified by the antecedent. The implication is done either by using Min function or Prod function (MATLAB help; Ross, 2010; Mohammadi \& Meech, 2013; Chen et al., 2011; Dağdeviren et al, 2009; Razani et al., 2013).

For the present study there are 30 rules in the rule base. There were $5,2 \& 3$ qualitative descriptor for FO, E \& CS consequently, based upon this the total number of rules become (No. of rules $-5 \times 2 \times 3)$.

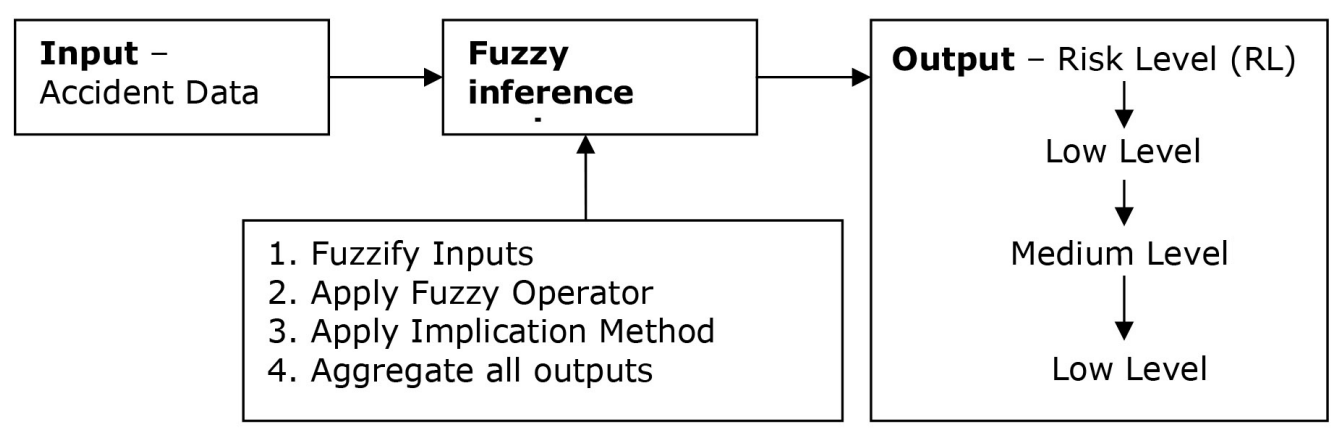

Figure 3. Fuzzy Inference System 
The fuzzy inference engine shown in Figure 3, will analyze input given to the system with the help of rule base developed and fed into the system. Thereafter the output will be given by the system after Defuzzification in terms of risk score. This score will be fit into the yardstick for risk levels and finally the level of risk associated with the hazard will be identified. The output obtained from FRA is lower level out. To get higher level output, the output of FRA is given as input to the FAHP system. This system will the final higher level output.

\subsection{Fuzzy Analytic Hierarchy Process (FAHP)}

FAHP determines weights by conducting pair wise comparison. pair wise comparison of sub criteria/hazard factors is done considering Saaty's 9 point scale (An et al., 2007; Mikhailov, 2004; Huang et al., 2005). This scale comprise of triangular fuzzy numbers to indicate intensity of importance. The scale is given below in Table 1.

\begin{tabular}{|l|l|c|}
\hline \multicolumn{1}{|c|}{ Intensity of importance in sub criteria } & \multicolumn{1}{c|}{ Explanation } & Triangular Fuzzy Numbers \\
\hline Equal Importance & Two hazard factor contribute equally & $(1,1,2)$ \\
\hline $\begin{array}{l}\text { Between equal importance and weak } \\
\text { importance }\end{array}$ & When compromise is needed & $(1,2,3)$ \\
\hline Weak importance & $\begin{array}{l}\text { Experience and judgment slightly favour } \\
\text { one hazard factor over another }\end{array}$ & $(3,4,4)$ \\
\hline Between weak and strong importance & When compromise is needed & $(4,5,6)$ \\
\hline Strong importance & $\begin{array}{l}\text { Experience and judgment strongly favour } \\
\text { one hazard factor over another }\end{array}$ & $(5,6,7)$ \\
\hline $\begin{array}{l}\text { Between strong and very strong } \\
\text { importance }\end{array}$ & \begin{tabular}{l} 
When compromise is needed \\
\hline Very strong importance
\end{tabular} & $\begin{array}{l}\text { An hazard factor is favoured very strongly } \\
\text { over another. }\end{array}$ \\
\hline $\begin{array}{l}\text { Between very strong and absolute } \\
\text { importance }\end{array}$ & When compromise is needed & $(6,7,8)$ \\
\hline Absolute importance & $\begin{array}{l}\text { One hazard factor over another is of the } \\
\text { highest possible affirmation }\end{array}$ & $(8,8,9)$ \\
\hline
\end{tabular}

Note: This scale is synthesized based on scheme suggested in (Huang et al., 2005; An et al., 2011; An et al., 2007; Chen et al., 2011).

Table 1. FAHP Scale 


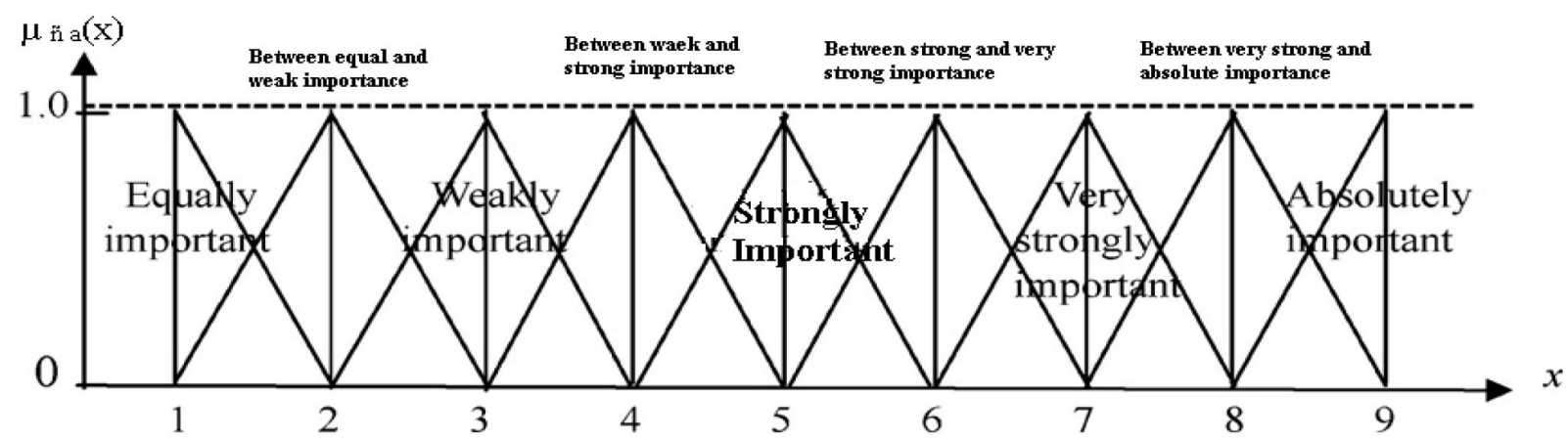

Figure 4. Membership function of linguistic variable for comparing two hazard factors/sub-criteria

The above mentioned scale is used to compare the sub criteria/hazard factors to know their degree of importance. Figure 4 shows membership function of linguistic variable for comparing the sub-criteria/hazard factors. Thereafter the FAHP matrix is prepared.

$$
\tilde{N}=\quad\left[\begin{array}{cccc} 
& & & \\
\tilde{n}_{1,1} & \tilde{n}_{1,2} & \tilde{n}_{1,3} \ldots \ldots . . & \tilde{n}_{1, n} \\
\tilde{n}_{2,1} & \tilde{n}_{2,2} & \tilde{n}_{2,3} \ldots \ldots \ldots & \tilde{n}_{2, n} \\
\tilde{n}_{3,1} & \tilde{n}_{3,2} & \tilde{n}_{3,3} \ldots \ldots \ldots & \tilde{n}_{3, n} \\
\tilde{n}_{n, 1} & \tilde{n}_{n, 2} & \tilde{n}_{n, 3} \ldots \ldots \ldots & \tilde{n}_{n, n}
\end{array}\right]
$$

Suppose there are two hazard factors/sub-criteria to be compared namely fall of roof and electricity. If fall of roof is absolutely important as compared to electricity, because once roof falls in underground mines number of people get affect and cases of electricity prone accidents are found less then based upon importance fall of roof will be assigned with fuzzy number $(9,9,9)$ and electricity will have fuzzy number $(1 / 9,1 / 9,1 / 9)$.

\subsubsection{Calculation of fuzzy weights and defuzzified weights}

To calculate fuzzy weights, arithmetic operations are performed on the fuzzy numbers. Triangular fuzzy number is a number with three points. For example $\tilde{n}_{a}\left(t_{a}^{l} t^{m}{ }_{a} t^{u}{ }_{a}\right)$ can be interpreted as-

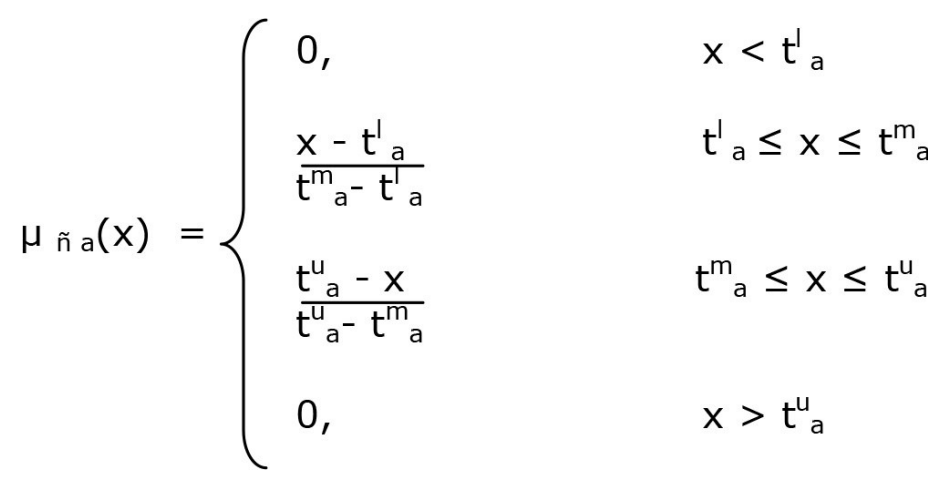


Where $t^{\prime}{ }_{a}$ and $t^{u}{ }_{a}$ stands for lower and upper bound of fuzzy number $\tilde{n}_{a}$, respectively, and $t^{m}{ }_{a}$ is the modal value. The fuzzy number can be denoted as $\tilde{n}_{a}\left(t_{a}^{\prime} t^{m}{ }_{a} t^{u}{ }_{a}\right)$. Below explained are the arithmetic operations performed on the two fuzzy numbers $\tilde{n}_{a}\left(t_{a}^{\prime} t^{m}{ }_{a} t^{u}{ }_{a}\right)$ and $\tilde{n}_{b}\left(t_{b}^{\prime} t^{m}{ }_{b} t_{b}^{u}\right)$. Membership function of triangular fuzzy number is shown in Figure 5.

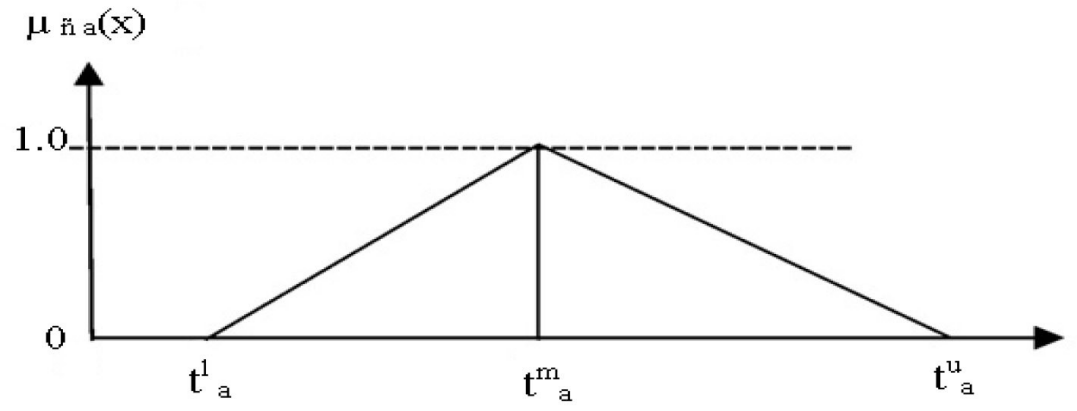

Figure 5. Membership Function of Triangular Fuzzy Number

The arithmetic operations that are performed on the two fuzzy numbers $\left\{\tilde{n}_{a}\left(t_{a}^{\prime} t^{m}{ }_{a} t^{u}{ }_{a}\right) \& \tilde{n}_{b}\left(t_{b}^{\prime}\right.\right.$ $\left.\left.t^{m}{ }_{b} t_{b}^{u}\right)\right\}$ are defined as follows (Ros, 2010; , An et al., 2011).

- Addition of a fuzzy number $\oplus$

$$
\tilde{n}_{a} \oplus \tilde{n}_{b}=\left(t_{a}^{\prime}+t_{b}^{\prime}, t^{m}{ }_{a}+t^{m}{ }_{b}, t^{u}{ }_{a}+t^{u}{ }_{b}\right)
$$

- Multiplication of a fuzzy number $\otimes$

$$
\tilde{n}_{a} \otimes \tilde{n}_{b}=\left(t_{a}^{1} \times t_{b}^{\prime}, t^{m}{ }_{a} \times t^{m}{ }_{b}, t^{u}{ }_{a} \times t^{u}{ }_{b}\right)
$$

- Division of a fuzzy number $\varnothing$

$$
\tilde{n}_{a} \varnothing \tilde{n}_{b}=\left(t_{a}^{\prime} / t^{u}{ }_{b}, t^{m}{ }_{a} / t^{m}{ }_{b}, t^{u}{ }_{a} / t_{b}^{\prime}\right)
$$

- Subtraction of a fuzzy number

$$
\tilde{n}_{a} \Theta \tilde{n}_{b}=\left(t_{a}^{\prime}-t^{u}{ }_{b}, t^{m}{ }_{a}-t^{m}{ }_{b}, t^{u}{ }_{a}-t_{b}^{\prime}\right)
$$

- Reciprocal of a fuzzy number

$$
\tilde{n}_{a}^{-1}=\left(t_{a}^{\prime} t^{m}{ }_{a} t_{a}^{u}\right)^{-1}=\left(1 / t_{a}^{\prime}, 1 / t^{m}{ }_{a}, 1 / t^{u}{ }_{a}\right)
$$

Where $\oplus, \otimes, \varnothing, \Theta$ stands for fuzzy logic addition, multiplication, division and subtraction operation respectively.

Fuzzy weights can be calculated by using geometric mean technique (Tang et al., 2000; Huang et al., 2005; Mikhailov, 2004; An et al., 2007; Saaty, 1980; Mohammadi \& Meech, 2013; Liu, Yang, Wang, Sii, 2004). 


$$
\begin{gathered}
f=\left(\tilde{n}_{i, 1} \otimes \tilde{n}_{i, 2} \otimes \ldots \tilde{n}_{i, n}\right)^{1 / n} \\
=\left(\left(t_{i, 1}^{1} \times t_{i, 2}^{1} \times t_{i, 3}^{1} \ldots \times t_{i, n}^{1}\right)^{1 / n},\right. \\
\left(t^{m_{i, 1}} \times t_{i, 2}^{m_{i, 2}} \times t^{m}{ }_{i, 3} \ldots \times t_{i, n}^{m}\right)^{1 / n}, \\
\left.\left(t^{u}{ }_{i, 1} \times t_{i, 2}^{u} \times t_{i, 3}^{u} \ldots \times t_{i, n}^{u}\right)^{1 / n}\right) \\
\tilde{w}_{i}=\frac{f_{i}}{f_{1} \oplus f_{2} \oplus f_{3} \oplus f_{4} \ldots f_{n}}
\end{gathered}
$$

$f_{i}=$ geometric mean of the $i^{\text {th }}$ row.

$\tilde{\mathrm{w}}_{\mathrm{i}}=$ fuzzy weight of the $\mathrm{i}^{\text {th }}$ event.

As soon as we get fuzzified weights of all the hazard factors, defuzzification of the weights is done, as the fuzzified weights are triangular fuzzy numbers and expected output is crisp in nature. The fuzzified weight obtained by geometric mean method is $\tilde{w}_{i}\left(t^{1}{ }_{i}, t^{m_{i}}, t^{u}\right)$, now, the defuzzified mean value of weight is calculated by (Tang et al., 2000; An et al., 2007; An et al., 2011; Mohammadi \& Meech, 2013).

$$
D F \tilde{w}_{i}=\frac{\left[\left(t^{u_{i}}-t^{\prime}{ }_{i}\right)+\left(t^{m_{i}-} t^{1}{ }_{i}\right)\right]}{3+t_{i}^{1}}
$$

Then $w_{i}$ is calculated as

$$
W_{i}=\frac{D F \tilde{W}_{i}}{\sum D F \tilde{W}_{i}}
$$

Overall risk at mine level can be calculated by the formula

$$
R L_{\text {overall }}=\Sigma R L_{i} W_{i}
$$

Where, $R_{i}$ is the risk score calculated from FRA system. To calculate overall risk level associated with the mine, $R_{i}$ for every identified hazard that caused mishap is calculated with the help of FRA system and then $R_{i}$ for the hazard is multiplied with $\tilde{w}_{i}$ of that particular hazard obtained from FAHP system and after summation of all these combined results of FRA and FAHP overall risk at mine level is obtained.

\section{Application of proposed approach}

For the present work the proposed integrated approach is used to evaluate levels of risk associated with hazards identified in metalliferrous mines. The mine selected for study extracts manganese and this mine is owned by central government undertaking organization, the mine 
is located in Maharashtra (India). The hazard factors were identified with the help of DGMS annual report (DGMS 2010, 2012) and for corresponding hazard factors accident data with consequence of severity, exposure level and frequency of occurrence from year 1995 to 2011 was gathered from the organization. With the analysis of accident data, the hazard factors/sub-criteria are identified as follows.

- Ground Movement

- Winding in shaft

- Transportation by machinery

- Machinery other than transportation

- Explosives,

- Electricity,

- Dust/gas,

- Falls other than ground

These hazard factors are evaluated to get an idea about the risk levels associated with them with the application of integrated approach. Thereafter overall mine level risk is evaluated so that the process of risk assessment can be adopted systematically and can help the safety managers to make effective mitigation plan on time which will ultimately lead to safe working environment.

\subsection{Application of FRA}

For the application of FRA approach Fuzzy Logic tool box of MATLAB is used. To build the Fuzzy Inference System in MATLAB for the case under study, fuzzy inputs were decided. Thereafter fuzzy operator is designated for antecedents of given rule if there is more then one part in antecedent in any rule. Two operators either AND or OR can be used for this purpose. Then implication method is applied before which the rule weight is decided. The weight of rule is between 0 to 1 , this weight is decided based upon the number given by the antecedent. The input for implication is the number provided by the antecedent and output of implication is a fuzzy set. After this output is aggregated. Aggregation is the process by which the fuzzy sets that represents the out puts of each rule are combined into a single fuzzy set. Then this fuzzy set is defuzzified and crisp output is obtained.

For the present case study a system named 'risk_assesment' is created, the type of system is 'mamdani' as it is most commonly seen fuzzy methodology and it is among first control 
systems built using fuzzy set theory (MATLAB help; Ros, 2010; Venkatrao, 2013). Number of inputs are 3 and output is 1 . Number of rules based on qualitative descriptor are 30 . For implication 'min' built-in-method is used and for defuzzification of the fuzzy set 'centroid' builtin-method is used.

The yardstick for the criteria and output along with membership function assigned to qualitative descriptor is given below in Table 2, 3, 4 and 5 (Verma \& Gupta, 2013) and Table 6 shows the rule base with rule antecedent, rule consequent, rule weight and connection, developed for fuzzy inference engine.

\begin{tabular}{|l|l|c|}
\hline \multicolumn{1}{|c|}{ Qualitative Descriptor } & \multicolumn{1}{c|}{ Description } & Parameters of MFs \\
\hline Extremely Infrequent & $5-10$ Years & $1.5,0$ (gaussian) \\
\hline Infrequent & $2-5$ years & $1.5,3.5$ (gaussian) \\
\hline Rather Frequent & $1-2$ Years & $4,5.5$ (gaussian) \\
\hline Frequent & 1 year & $6,7.5$ (gaussian) \\
\hline Very Frequent & Accident/month & 8,10 (gaussian) \\
\hline
\end{tabular}

Table 2. Frequency of Occurrence/Probability of Accidents (FO)

\begin{tabular}{|l|l|l|}
\hline \multicolumn{1}{|c|}{ Qualitative Descriptor } & \multicolumn{1}{|c|}{ Description } & Parameters Of Mfs \\
\hline Reportable & Minor Injury & $1.5,0$ (gaussian) \\
\hline Serious & Major Injury & $1.5,5$ (gaussian) \\
\hline Fatal & Death & 5,10 (gaussian)) \\
\hline
\end{tabular}

Table 3. Consequence of Severity (CS)

\begin{tabular}{|l|l|c|}
\hline \multicolumn{1}{|c|}{ Qualitative Descriptor } & \multicolumn{1}{|c|}{ Description } & Parameters Of Mfs \\
\hline Low Level & One Person getting exposed to the hazard & $0,0,1,3$ (trapezoid) \\
\hline High Level & More than one person getting exposed to the hazard & $7,9,10,10$ (trapezoid) \\
\hline
\end{tabular}

Table 4. Exposure Level (E)

\begin{tabular}{|l|l|c|c|}
\hline \multicolumn{1}{|c|}{ Qualitative Descriptor } & \multicolumn{1}{|c|}{ Description } & \multicolumn{1}{c|}{ Risk } & Parameters of Mfs \\
\hline High & $\begin{array}{l}\text { Risk must be reduced safe in } \\
\text { exceptional circumstances. }\end{array}$ & $200<=$ risk score $<=500$ & $\begin{array}{c}300,400,500 \\
\text { (triangular) }\end{array}$ \\
\hline Medium & $\begin{array}{l}\text { Risk must be reduced if it is } \\
\text { reasonably practicable to do so. }\end{array}$ & $20<=$ risk score $<=200$ & $\begin{array}{c}150,200,300 \\
\text { (triangular) }\end{array}$ \\
\hline Low & Risk is acceptable & $20>$ risk score & $\begin{array}{c}0,50,100 \\
\text { (triangular) }\end{array}$ \\
\hline
\end{tabular}

Table 5. Risk Level (RL) 


\begin{tabular}{|c|c|c|c|c|c|}
\hline \multicolumn{3}{|c|}{ Rule Antecedent } & \multirow{2}{*}{$\begin{array}{c}\text { Rule Consequent } \\
1 \\
\end{array}$} & \multirow{2}{*}{$\begin{array}{c}\text { Rule Weight } \\
(1)\end{array}$} & \multirow{2}{*}{$\begin{array}{c}\text { Rule Connection } \\
1 \\
\end{array}$} \\
\hline 1 & 1 & 1 & & & \\
\hline 1 & 1 & 2 & 2 & $(1)$ & 1 \\
\hline 1 & 1 & 1 & 3 & (1) & 1 \\
\hline 1 & 2 & 1 & 1 & (1) & 1 \\
\hline 1 & 2 & 2 & 2 & (1) & 1 \\
\hline 1 & 2 & 3 & 3 & (1) & 1 \\
\hline 1 & 3 & 1 & 1 & $(1)$ & 1 \\
\hline 1 & 3 & 2 & 2 & (1) & 1 \\
\hline 1 & 3 & 3 & 3 & (1) & 1 \\
\hline 1 & 4 & 1 & 1 & (1) & 1 \\
\hline 1 & 4 & 2 & 3 & (1) & 1 \\
\hline 1 & 4 & 3 & 3 & $(1)$ & 1 \\
\hline 1 & 5 & 1 & 3 & (1) & 1 \\
\hline 1 & 5 & 2 & 3 & (1) & 1 \\
\hline 1 & 5 & 3 & 3 & (1) & 1 \\
\hline 2 & 1 & 1 & 1 & (1) & 1 \\
\hline 2 & 1 & 2 & 2 & $(1)$ & 1 \\
\hline 2 & 1 & 3 & 3 & (1) & 1 \\
\hline 2 & 2 & 1 & 1 & (1) & 1 \\
\hline 2 & 2 & 2 & 3 & (1) & 1 \\
\hline 2 & 2 & 3 & 3 & (1) & 1 \\
\hline 2 & 3 & 1 & 2 & (1) & 1 \\
\hline 2 & 3 & 2 & 3 & (1) & 1 \\
\hline 2 & 3 & 3 & 3 & (1) & 1 \\
\hline 2 & 4 & 1 & 3 & (1) & 1 \\
\hline 2 & 4 & 2 & 3 & (1) & 1 \\
\hline 2 & 4 & 3 & 3 & (1) & 1 \\
\hline 2 & 5 & 1 & 3 & (1) & 1 \\
\hline 2 & 5 & 2 & 3 & (1) & 1 \\
\hline 2 & 5 & 3 & 3 & (1) & 1 \\
\hline
\end{tabular}

Table 6. Rule base

The accident data is evaluated based on the above mentioned three criteria using fuzzy logic tool box of Matlab software and the output which is the risk score for the hazard factor is obtained. The risk scores for all the hazard factors are given below in the Table 7:

\begin{tabular}{|l|c|l|l|}
\hline Hazard Factor & Risk Score $\left(\mathbf{R L}_{\mathbf{i}}\right)$ & \multicolumn{1}{|c|}{ Risk Level } & \multicolumn{1}{|l|}{ Membership Functions (Mfs) } \\
\hline Ground Movement & 321.75 & High Level Risk & $300,400,500$ (triangular) \\
\hline Winding in Shaft & 286.76 & Medium Level Risk & $150,200,300$ (triangular) \\
\hline Transportation by Machinery & 286.76 & Medium Level Risk & $150,200,300$ (triangular) \\
\hline Machinery other than transportation & 50 & Low Level Risk & $0,50,100$ (triangular) \\
\hline Explosives & 50 & Low Level Risk & $0,50,100$ (triangular) \\
\hline Electricity & 286.76 & Medium Level Risk & $150,200,300$ (triangular) \\
\hline Dust/Gas & 41.5 & Low Level Risk & $0,50,100$ (triangular) \\
\hline Falls other than grounds & 321.37 & High Level Risk & $300,400,500$ (triangular) \\
\hline
\end{tabular}

Table 7. Risk Level of Hazards obtained from FRA 
The output of the above described approach is risk score which is fit in the yardstick of risk level to know the level of risk associated with the hazard. As shown above the risk score for ground movement is 321.75 , this score fits within the range specified for the qualitative descriptor i.e. high level risk in the yardstick for risk level. Similarly remaining for all the hazard factors risk scores are interpreted.

The output of FRA approach as discussed is low level output and contains uncertainty, to filter out the uncertainty and to get crisp output; the output of FRA is taken as input for FAHP approach.

\subsection{Application of FAHP approach}

FAHP analysis starts with the development of pair wise comparison matrix. For the present study the pair wise comparison matrix is for hazard factor is developed, which is shown below in Table 8:

\begin{tabular}{|c|c|c|c|c|c|c|c|c|}
\hline Index & $\mathbf{1}$ & $\mathbf{2}$ & $\mathbf{3}$ & $\mathbf{4}$ & $\mathbf{5}$ & $\mathbf{6}$ & $\mathbf{7}$ & $\mathbf{8}$ \\
\hline 1 & $1,1,1$ & $8,9,9$ & $6,7,8$ & $8,9,9$ & $8,9,9$ & $8,9,9$ & $5,6,7$ & $5,6,7$ \\
\hline 2 & $\begin{array}{c}0.11,0.11, \\
0.13\end{array}$ & $1,1,1$ & $1,0.5,0.33$ & $1,2,2$ & $3,4,5$ & $3,4,5$ & $\begin{array}{c}0.13,0.11, \\
0.11\end{array}$ & $\begin{array}{c}0.13,0.11, \\
0.11\end{array}$ \\
\hline 3 & $\begin{array}{c}0.13,0.14, \\
0.17\end{array}$ & $3,2,1$ & $1,1,1$ & $4,5,6$ & $5,6,7$ & $5,6,7$ & $\begin{array}{c}0.13,0.11, \\
0.11\end{array}$ & $\begin{array}{c}0.13,0.11, \\
0.11\end{array}$ \\
\hline 4 & $\begin{array}{c}0.11,0.11, \\
0.13\end{array}$ & $0.5,1,1$ & $\begin{array}{c}0.17,0.2, \\
0.25\end{array}$ & $1,1,1$ & $4,5,6$ & $4,5,6$ & $\begin{array}{c}0.13,0.11, \\
0.11\end{array}$ & $\begin{array}{c}0.13,0.11, \\
0.11\end{array}$ \\
\hline 5 & $\begin{array}{c}0.11,0.11, \\
0.13\end{array}$ & $\begin{array}{c}0.2,0.25, \\
0.33\end{array}$ & $\begin{array}{c}0.17,0.2,0 . \\
25\end{array}$ & $\begin{array}{c}0.17,0.2, \\
0.25\end{array}$ & $1,1,1$ & $1,1,2$ & $\begin{array}{c}0.13,0.11, \\
0.11\end{array}$ & $\begin{array}{c}0.13,0.11, \\
0.11\end{array}$ \\
\hline 6 & $\begin{array}{c}0.11,0.11, \\
0.13\end{array}$ & $\begin{array}{c}0.2,0.25, \\
0.33\end{array}$ & $\begin{array}{c}0.17,0.2, \\
0.25\end{array}$ & $\begin{array}{c}0.17,0.2, \\
0.25\end{array}$ & $0.5,1,1$ & $1,1,1$ & $\begin{array}{c}0.13,0.11, \\
0.11\end{array}$ & $\begin{array}{c}0.13,0.11, \\
0.11\end{array}$ \\
\hline 7 & $\begin{array}{c}0.14,0.17, \\
0.2\end{array}$ & $9,9,8$ & $0.11,0.11$, & $9,9,8$ & $9,9,8$ & $9,9,8$ & $1,1,1$ & $1,1,2$ \\
\hline 8 & $\begin{array}{c}0.14,0.17, \\
0.2\end{array}$ & $9,9,8$ & $0.11,0.11$, & $9,9,8$ & $9,9,8$ & $9,9,8$ & $2,1,1$ & $1,1,1$ \\
\hline
\end{tabular}

Table 8. Pair wise comparison matrix

Where,

$1=$ Ground movement

$2=$ Winding in shaft

$3=$ Transportation by machinery

$4=$ Machinery other than transportation

$5=$ Explosives 
6 = Electricity

$7=$ Dust $/$ Gas

$8=$ Falls other than ground

\subsubsection{Assessment of priority weights for the hazard factors/sub-criteria}

FAHP analysis starts with calculation of weight associated with hazards on the basis of Equation 6 and 7. On the basis of these equations the geometric mean technique gives the fuzzy weight of the hazards factors.

Step 1. Calculation of fuzzy weights

$$
\begin{aligned}
& f=\left(\tilde{n}_{i, 1} \otimes \tilde{n}_{i, 2} \otimes \ldots \tilde{n}_{i, n}\right)^{1 / n} \\
& =\left(\left(t_{i, 1}^{1} \times t_{i, 2}^{1} \times t_{i, 3}^{1} \ldots \times t_{i, n}^{1}\right)^{1 / n},\right. \\
& \left(t^{m_{i, 1}} \times t^{m_{i, 2}} \times t^{m_{i, 3}} \ldots \times t^{m}{ }_{i, n}\right)^{1 / n} \text {, } \\
& \left.\left(\mathrm{t}^{\mathrm{u}}{ }_{\mathrm{i}, 1} \times \mathrm{t}_{\mathrm{i}, 2}^{\mathrm{u}} \times \mathrm{t}_{\mathrm{i}, 3}^{\mathrm{u}} \ldots \times \mathrm{t}_{\mathrm{i}, \mathrm{n}}^{\mathrm{u}}\right)^{1 / \mathrm{n}}\right) \\
& f_{1}=\left[(1 \times 8 \times 6 \times 8 \times 8 \times 8 \times 5 \times 5)^{1 / 8},(1 \times 9 \times 7 \times 9 \times 9 \times 9 \times 6 \times 6)^{1 / 8},(1 \times 9 \times 8 \times 9 \times 9 \times 9 \times 7 \times 7)^{1 / 8}\right] \\
& f_{1}=(5.29,5.98,6.32)
\end{aligned}
$$

Similarly, Table 9 shows geometric mean $\left(f_{i}\right)$ value for all hazard factors.

\begin{tabular}{|c|c|}
\hline$f_{1}=(5.29,5.98,6.32)$ & $f_{5}=(0.23,0.24,0.29)$ \\
\hline$f_{2}=(0.60,0.60,0.63)$ & $f_{6}=(0.21,0.24,0.27)$ \\
\hline$f_{3}=(0.94,0.94,0.93)$ & $f_{7}=(1.78,1.8,1.95)$ \\
\hline$f_{4}=(0.47,0.53,0.58)$ & $f_{8}=(1.94,1.82,1.79)$ \\
\hline
\end{tabular}

Table 9. value of geometric mean $\left(f_{i}\right)$ for all hazard factors

$$
\tilde{w}_{i}=\frac{\mathcal{f}_{i}}{f_{1} \oplus f_{2} \oplus f_{3} \oplus f_{4} \ldots f_{n}}
$$

$(5.29,5.98,6.32)$

$$
\begin{aligned}
\tilde{w}_{1}= & \frac{(5.29,5.98,6.32) \oplus(0.60,0.60,0.63) \oplus(0.94,0.94,0.93) \oplus(0.47,0.53,}{0.58)(0.23,0.24,0.29) \oplus(0.21,0.24,0.27) \oplus(1.78,1.8,1.95) \oplus(1.94,1.82,1.79)}
\end{aligned}
$$

Similarly, Table 10 shows the value of fuzzy weights $\left(\tilde{w}_{i}\right)$ for all hazard factors. 


\begin{tabular}{|l|l|}
\hline$\tilde{\mathrm{W}}_{1}=(0.41,0.47,0.55)$ & $\tilde{\mathrm{W}}_{5}=(0.01,0.01,0.02)$ \\
\hline$\tilde{\mathrm{W}}_{2}=(0.04,0.05,0.05)$ & $\tilde{\mathrm{W}}_{6}=(0.01,0.01,0.02)$ \\
\hline$\tilde{\mathrm{W}}_{3}=(0.07,0.07,0.08)$ & $\tilde{\mathrm{W}}_{7}=(0.13,0.14,0.17)$ \\
\hline$\tilde{\mathrm{W}}_{4}=(0.03,0.04,0.05)$ & $\tilde{\mathrm{W}}_{8}=(0.15,0.15,0.16)$ \\
\hline
\end{tabular}

Table 10. value of fuzzy weights $\left(\tilde{W}_{i}\right)$ of hazard factors

Step 2. Defuzzification of the weights

Fuzzy priority weights calculated above are defuzzified to get crisp weights using Equation 8.

DF $\tilde{W}_{i}=$ defuzzified mean value of weight

$$
\begin{gathered}
\text { DF } \tilde{w}_{i}=\frac{\left[\left(t^{u_{i}}-t_{i}^{1}\right)+\left(t^{m_{i}}-t_{i}^{\prime}\right)\right]}{3+t_{i}^{1}} \\
\tilde{w}_{1}=(0.41,0.47,0.55) \\
\text { DF } \tilde{w}_{1}=0.058
\end{gathered}
$$

Similarly, Table 11 shows defuzzified mean value of weights of all the hazard factors/subcriteria.

\begin{tabular}{|l|l|}
\hline DF $\tilde{\mathrm{w}}_{1}=0.058$ & DF $\tilde{\mathrm{w}}_{5}=0.003$ \\
\hline DF $\tilde{\mathrm{w}}_{2}=0.006$ & DF $\tilde{\mathrm{w}}_{6}=0.006$ \\
\hline DF $\tilde{\mathrm{w}}_{3}=0.003$ & DF $\tilde{\mathrm{w}}_{7}=0.015$ \\
\hline DF $\tilde{\mathrm{w}}_{4}=0.009$ & DF $\tilde{\mathrm{w}}_{8}=0.003$ \\
\hline
\end{tabular}

Table 11. defuzzified mean value of weights of hazard factors/sub-criteria

Step 3. Normalization of defuzzied weights

Normalization of the defuzzified weights is done using Equation 9.

Where,

$$
\begin{aligned}
& \Sigma \mathrm{DF} \tilde{\mathrm{w}}_{i}=0.103 \\
& \mathrm{w}_{i}=\frac{\mathrm{DF} \tilde{\mathrm{W}}_{\mathrm{i}}}{\Sigma \mathrm{DF} \tilde{\mathrm{w}}_{\mathrm{i}}}
\end{aligned}
$$

Now, 


$$
\begin{gathered}
\mathrm{w}_{1}=\frac{0.058}{0.103} \\
\mathrm{w}_{1}=0.56
\end{gathered}
$$

Similarly, Table 12 shows normalized defuzzified weights of all the hazard factors/sub-criteria.

\begin{tabular}{|c|c|}
\hline $\mathrm{w}_{1}=0.56$ & $\mathrm{w}_{5}=0.02$ \\
\hline $\mathrm{w}_{2}=0.05$ & $\mathrm{w}_{6}=0.05$ \\
\hline $\mathrm{w}_{3}=0.02$ & $\mathrm{w}_{7}=0.145$ \\
\hline $\mathrm{w}_{4}=0.08$ & $\mathrm{w}_{8}=0.02$ \\
\hline
\end{tabular}

Table 12. normalized defuzzified weights of hazard factors/sub-criteria

Step 4. Overall risk estimation

Overall risk estimation at mine level can be calculated using Equation 10.

$$
\begin{gathered}
\mathrm{RL}_{\text {overall }}=\sum \mathrm{RL}_{\mathrm{i}} \mathrm{w} \\
\mathrm{RL}_{1}=\mathrm{W}_{1} \times \text { FRA risk score for hazard } 1 \\
=0.56 \times 321.75 \\
\mathrm{RL}_{1}=180.18
\end{gathered}
$$

Similarly, Table 13 shows the FRA risk score of all the hazard factors.

\begin{tabular}{|c|c|}
\hline $\mathrm{RL}_{1}=180.18$ & $\mathrm{RL}_{5}=1$ \\
\hline $\mathrm{RL}_{2}=2.5$ & $\mathrm{RL}_{6}=2.5$ \\
\hline $\mathrm{RL}_{3}=5.73$ & $\mathrm{RL}_{7}=41.5$ \\
\hline $\mathrm{RL}_{4}=22.94$ & $\mathrm{RL}_{8}=6.42$ \\
\hline
\end{tabular}

Table 13. FRA risk score of hazard factors/sub-criteria

From the RL values calculated above the hazard with maximum risk associated can be identified and accordingly based on priority basis mitigation plan to treat the hazard can be prepared. In the present case looking at the $\mathrm{RL}_{s}$, the priority ranking of hazard is-

$$
\mathrm{RL}_{1}>\mathrm{RL}_{7}>\mathrm{RL}_{4}>\mathrm{RL}_{8}>\mathrm{RL}_{3}>\mathrm{RL}_{2}>\mathrm{RL}_{6}>\mathrm{RL}_{5}
$$


or

$$
\mathrm{RL}_{1}>\mathrm{RL}_{7}>\mathrm{RL}_{4}>\mathrm{RL}_{8}>\mathrm{RL}_{3}>\mathrm{RL}_{6}>\mathrm{RL}_{2}>\mathrm{RL}_{5}
$$

Using the Equation 10 overall risk in any of the mining site can be calculated as

$$
\begin{aligned}
\mathrm{RL}_{\text {overall }} & =\sum R \mathrm{~L}_{i} \\
\mathrm{RL}_{\text {overall }} & =262.77
\end{aligned}
$$

The above shown risk score is fit in the yardstick of risk level and it is observed that the level

\begin{tabular}{|c|c|c|c|c|c|}
\hline $\boldsymbol{R}_{\boldsymbol{i}}$ & $\boldsymbol{f}_{i}$ & $\tilde{w}_{i}$ & $D F \tilde{w}_{i}$ & $w_{i}$ & $\boldsymbol{R} \boldsymbol{L}_{i} \boldsymbol{W}_{i}$ \\
\hline 321.75 & $f_{1}=(5.29,5.98,6.32)$ & $\tilde{\mathrm{w}}_{1}=(0.41,0.47,0.55)$ & $\mathrm{DF} \tilde{\mathrm{w}}_{1}=0.058$ & $w_{1}=0.56$ & $\mathrm{RL}_{1}=180.18$ \\
\hline 286.76 & $f_{2}=(0.60,0.60,0.63)$ & $\tilde{\mathrm{w}}_{2}=(0.04,0.05,0.05)$ & $\mathrm{DF} \tilde{\mathrm{w}}_{2}=0.006$ & $w_{2}=0.05$ & $\mathrm{RL}_{2}=2.5$ \\
\hline 286.76 & $f_{3}=(0.94,0.94,0.93)$ & $\tilde{\mathrm{w}}_{3}=(0.07,0.07,0.08)$ & $\mathrm{DF} \tilde{\mathrm{w}}_{3}=0.003$ & $w_{3}=0.02$ & $\mathrm{RL}_{3}=5.73$ \\
\hline 50 & $f_{4}=(0.47,0.53,0.58)$ & $\tilde{\mathrm{W}}_{4}=(0.03,0.04,0.05)$ & DF $\tilde{\mathrm{W}}_{4}=0.009$ & $\mathrm{w}_{4}=0.08$ & $\mathrm{RL}_{4}=22.94$ \\
\hline 50 & $f_{5}=(0.23,0.24,0.29)$ & $\tilde{w}_{5}=(0.01,0.01,0.02)$ & $\mathrm{DF} \tilde{\mathrm{W}}_{5}=0.003$ & $W_{5}=0.02$ & $R L_{5}=1$ \\
\hline 286.76 & $f_{6}=(0.21,0.24,0.27)$ & $\tilde{\mathrm{W}}_{6}=(0.01,0.01,0.02)$ & DF $\tilde{\mathrm{w}}_{6}=0.006$ & $\mathrm{w}_{6}=0.05$ & $\mathrm{RL}_{6}=2.5$ \\
\hline 286.76 & $f_{7}=(1.78,1.8,1.95)$ & $\tilde{\mathrm{w}}_{7}=(0.13,0.14,0.17)$ & $\mathrm{DF} \tilde{\mathrm{w}}_{7}=0.015$ & $W_{7}=0.145$ & $\mathrm{RL}_{7}=41.5$ \\
\hline 321.37 & $f_{8}=(1.94,1.82,1.79)$ & $\tilde{\mathrm{w}}_{8}=(0.15,0.15,0.16)$ & $\mathrm{DF} \tilde{\mathrm{w}}_{8}=0.003$ & $\mathrm{w}_{8}=0.02$ & $\mathrm{RL}_{8}=6.42$ \\
\hline
\end{tabular}
of risk associated at mine level is medium.

\subsubsection{Results and discussions}

Table 14. Final Results of FAHP approach

Table 14 shows the result obtained from the application of FAHP approach. The overall RL obtained is 262.77 . This score is fit in the yardstick of risk level to conclude that the risk associated at mine level for all the operations performed to extract ore from ore body is medium. The summary of analysis indicates the risk level associated with individual hazard, like the output obtained from FRA shown above in terms of $R_{i}$ gives preliminary idea that maximum risk is associated with hazard ground movement with maximum risk score of 321.75 followed by the hazard falls other than ground with risk score of 321.37. After combining the results of FRA with AHP, the final conclusion about the risk levels of hazard are drawn, the most risky hazard having maximum potential to create unacceptable consequences is ground movement with $\mathrm{RL}=180.18$ followed by dust hazard with $\mathrm{RL}=41.5$, similarly conclusions can be drawn about every identified hazard based upon RL obtained from FAHP. After getting priority risk scores for the hazards, the mitigation plan for the hazard is prepared accordingly so that precautionary actions can be taken for most risky hazard on priority basis and workplace safety can be improved. 


\section{Conclusion}

This paper proposed a novel approach which is integration of FRA and AHP approaches for evaluation of risk levels associated with identified hazard factors in mining industry. The traditional approach adopted for risk assessment in the mining industry relies upon availability and accuracy of data. Traditional technique is not sufficient enough to deal with uncertain data. To deal with such problem, proposed approach can be adopted. FRA approach can efficiently manage uncertain input data for the system and to get crisp and robust output from the system without any uncertainty, the output obtained from FRA approach is combined with AHP technique. The proposed model gives output in terms of RLs associated with hazard factors identified and finally after combining the risk scores of all the hazard factors the overall risk associated with the mining site is obtained. The proposed model can be adopted for systematic assessment of risk in the said industry as risk assessment is a requirement of the Occupational Health and Safety Act 2000 (section $7 \&$ 8). The advantages of the proposed risk assessment model can be summarized as: (1) it can handle expert knowledge, engineering judgments. (2) it is efficient enough to deal with uncertain and imprecise data (3) as the approach is integrated and hybridized the uncertainty in output gets filtered and robust final output is obtained (4) as the approach is systematic and structured it is not required to start always from scratch, any hazard factor can be analyzed based upon mentioned criteria. This approach will help decision makers, risk analyst, safety managers to make plan for mitigation of the risky hazards and develop safe work environment.

\section{References}

An, M., Huang, S. \& Baker, C.J. (2007). Railway risk assessment-the fuzzy reasoning approach and fuzzy analytic hierarchy process approaches: A case study of shunting at waterloo depot. Proc, ImechE, Part F: J. Rail and Rapid Transit, 221, 365-382.

http://dx.doi.org/10.1243/09544097JRRT106

An, M., Chen, Y., \& Baker, C.J. (2011). A fuzzy reasoning and fuzzy-analytical hierarchy process based approach to the process of railway risk information: A railway risk management system. Information Sciences, 181, 3946-3966. http://dx.doi.org/10.1016/j.ins.2011.04.051

Buyukozkan, G., \& Cifci, G. (2012a). A novel integrated MCDM approach based on fuzzy DEMATEL, fuzzy ANP and fuzzy TOPSIS to evaluate green suppliers. Expert Systems with Applications, 39, 3000-3011. http://dx.doi.org/10.1016/j.eswa.2011.08.162

Buyukozkan, G., \& Cifci, G. (2012b). A combined fuzzy AHP and fuzzy TOPSIS based strategic analysis of electronic service quality in healthcare industry. Expert Systems with Applications, 39, 2341-2354. http://dx.doi.org/10.1016/j.eswa.2011.08.061 
Chen, J., \& Yang, Y. (2011). A fuzzy ANP based approach to evaluate region agricultural drought risk. Procedia Engineering, 23, 822-827. http://dx.doi.org/10.1016/j.proeng.2011.11.2588

Chen, V.Y.C., Lien, H-P., Liu, C-H., Liou, J.J.H., Tzeng, G-H., Yang, L-S. (2011). Fuzzy MCDM approach for selecting the best environment-watershed plan. Applied Soft Computing, 11, 265-275. http://dx.doi.org/10.1016/j.asoc.2009.11.017

Coyle G. (2004). The analytic hierarchy process (AHP). Pearson education limited.

Dağdeviren, M., Yavuz, S., \& Kılınç, N. (2009). Weapon selection using the AHP and TOPSIS methods under fuzzy environment. Expert Systems with Applications, 36, 8143-8151. http://dx.doi.org/10.1016/j.eswa.2008.10.016

Directorate General of Mining Safety (2012). Annual Report.

Directorate General of Mining Safety (2010). Final report on Health and Safety Management System of An U/G Mine. Prepared by Society for Mining research, Sustainable Development and Environment Kolkata, 1/2.

Huang, S., An, M., \& Baker, C.J. (2005). A fuzzy based approach to risk assessment for track maintenance incorporated with AHP. In proceedings of International Railway Engineering conference, London, 29-30 Nov. Engineering Technics Press, Edinburgh.

Khalil, M., Abdou, M.A., Mansour, M.S., Farag, H.A., \& Ossman, M.E. (2012). A cascaded fuzzyLOPA risk assessment model applied in natural gas industry. Journal of Loss Prevention in the Process Industries, 25, 877-882. http://dx.doi.org/10.1016/j.jp.2012.04.010

Lakshminarayan, V., \& Singh, D. (2000). Risk Management as a tool to improve Safety in Mines. National Seminar on Specific Problems in Mining both Coal and Non-Coal Mines held at Puri.

Liu, J., Yang, J-B., Wang, J., Sii, H.S., \& Wang, Y.M. (2004). Fuzzy rule-based evidential reasoning approach for safety analysis. International Journal of General Systems, 33(2-3), 183-204. http://dx.doi.org/10.1080/03081070310001633536

MATLAB help for fuzzy logic toolbox.

Mikhailov, L. (2004). A fuzzy approach to deriving priorities from interval pairwise comparison judgements. Eur. J. Oper. Res, 159(3), 687-704. http://dx.doi.org/10.1016/S0377-2217(03)00432-6

Mohammadi, L., \& Meech, J.A. (2013). AFRA Heuristic expert system to assess the atmospheric risk of sulphide waste dumps. Journal of Loss Prevention in the Process Industries, 26, 261-271. http://dx.doi.org/10.1016/j.jp.2012.11.009

Paliwal, R., \& Jain, M.L. (2001). Assessment \& Management of Risk- An Overview. The Indian Mining And Engineering Journal.

Pathak, K., \& Sen, P. (2001). Risk Assessment in Mining Industry. The Indian Mining And Engineering Journal. 
Razani, M., Yazdani-Chamzini, A., \& Yakhchali, S.H. (2013). A novel fuzzy inference system for predicting roof fall rate in underground coal mines. Safety Science, 55, 26-33. http://dx.doi.org/10.1016/j.ssci.2012.11.008

Ross, T.J. (2010). Fuzzy Logic with Engineering Application. Second edition. Wiley Publication. http://dx.doi.org/10.1002/9781119994374

Rouhani, S., Ghazanfari, M., \& Jafari, M. (2012). Evaluation model of business intelligence for enterprise systems using fuzzy TOPSIS. Expert Systems with Applications, 39, 3764-3771. http://dx.doi.org/10.1016/j.eswa.2011.09.074

Saaty, T.L. (1980). Analytical Hierarchy Process. New York: McGraw-Hill.

Tang, M.T., Tzeng, G.H., \& Wang, S.W. (2000). A Hierarchy fuzzy MCDM method for studying electronic marketing strategies in the information service industry. J. Int. Inf. Manage, 8(1), 1-22.

Tripathy, D.P., \& Patra, A.K. (1998). Safety Risk Assessment In Mines. The Indian Mining And Engineering Journal.

Vahdani, B., Mousavi, S.M. \& Tavakkoli-Moghaddam, R. (2011). Group decision making based on novel fuzzy modified TOPSIS method. Applied mathematics modeling, 35, 4257-4269. http://dx.doi.org/10.1016/j.apm.2011.02.040

Venkatrao, R. (2013). Decision making in the manufacturing environment using graphical theory and fuzzy multiple attribute decision making methods. Springer.

Verma, S., \& Gupta, M. (2013). Risk assessment in mining industry. International Journal of Mining and Mineral Engineering, 4(4), 312-331. http://dx.doi.org/10.1504/IJMME.2013.056857

Wang, J., Fan, K., \& Wang, W. (2010). Integration of fuzzy AHP and FPP with TOPSIS methodology for aeroengine health assessment. Expert Systems with Applications, 37, 8516-8526. http://dx.doi.org/10.1016/j.eswa.2010.05.024

Wang, Y.J., \& Lee, H.S. (2007). Generalizing TOPSIS for fuzzy multiple-criteria group decisionmaking. Computers and mathematics with applications, 53, 1762-1772.

http://dx.doi.org/10.1016/j.camwa.2006.08.037

Journal of Industrial Engineering and Management, 2014 (www.jiem.org)

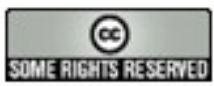

Article's contents are provided on a Attribution-Non Commercial 3.0 Creative commons license. Readers are allowed to copy, distribute and communicate article's contents, provided the author's and Journal of Industrial Engineering and Management's names are included. It must not be used for commercial purposes. To see the complete license contents, please visit http://creativecommons.org/licenses/by-nc/3.0/. 